\title{
Association of joint awareness with satisfaction, knee function, and noise perception after total knee arthroplasty.
}

Hiroto Taniguchi

Tokyo Joshi Ika Daigaku Yachiyo Iryo Center

Masafumi Itoh

Tokyo Joshi lka Daigaku

Nobuyuki Yoshimoto

Tokyo Joshi lka Daigaku Yachiyo Iryo Center

Junya Itou

Tokyo Joshi Ika Daigaku Byoin

Umito Kuwashima

Tokyo Joshi Ika Daigaku Byoin

Ken - Okazaki ( $\square$ okazaki.ken@twmu.ac.jp )

Tokyo Joshi lka Daigaku https://orcid.org/0000-0003-1274-8406

Research article

Keywords: total knee arthroplasty; patient-reported outcome measures; joint awareness; patient satisfaction; noise

Posted Date: August 5th, 2019

DOI: https://doi.org/10.21203/rs.2.12104/v1

License: (9) This work is licensed under a Creative Commons Attribution 4.0 International License.

Read Full License 


\section{Abstract}

Background: Forgotten Joint Score-12 (FJS-12) measures the clinical outcomes focusing on joint awareness after surgery, such as total knee arthroplasty (TKA). The Knee Society Scoring System-2011 (KSS-2011) includes questionnaires for satisfaction, expectation, and functional activities. However, the relationship between FJS-12 and KSS-2011 has not been validated to our knowledge. In addition, some patients complain of noise after TKA. However, how the noise affects joint awareness and satisfaction has not been evaluated. The aim of this study is to clarify the relationship among FJS-12, KSS-2011 and the noise. Methods: Using the FJS-12 and KSS-2011, 295 knees from 225 patients who underwent TKA was retrospectively evaluated. Noise perception was evaluated by a questionnaire with five grades which is a method that follows the questionnaire form of FJS-12 ["Are you aware of the noise of your artificial joint?" ; never, almost never, seldom, sometimes, mostly]. The correlations among FJS-12, KSS-2011, and noise were analyzed. In addition, patients were divided into four groups based on the mechanism of their implant [cruciate retaining, posterior stabilized, cruciate sacrificed, and bicruciate stabilized (BCS)]. FJS12 KSS-2011, and noise were compared among the groups. Results: A strong correlation was observed between FJS-12 and the total score of KSS-2011 (0.70; P < 0.001). FJS-12 also was correlated with KSS2011 subcategories for "symptoms," "satisfaction," and "standard activities" that these correlation coefficients were approximately 0.60 . Noise was limited to have weak correlations with FJS-12 $(0.28 ; \mathrm{P}<$ $0.001)$ and KSS-2011 (0.20 P < 0.001). With comparison among the TKA mechanisms, BCS showed significantly better KSS-2011 and greater range of movement but worse noise scores. Conclusions: Joint awareness assessed by FJS-12 was correlated strongly with KSS-2011 and associated with satisfaction, residual symptoms, and daily activities as assessed by KSS-2011 subscores. Noise perception had limited effect on joint awareness and clinical outcomes.

\section{Background}

Total knee arthroplasty (TKA) is a standard surgical treatment for advanced stage knee osteoarthritis [1]. Regarding the method used to evaluate its outcome, physician-derived scores had an important role in the past. However, an inconsistency was observed between physician- and patient-derived scores [2]. In addition, several reports show that patient satisfaction after TKA is not high [3-5]. Therefore, patientreported outcome measures (PROMs), such as the Western Ontario and McMaster Universities Osteoarthritis Index (WOMAC) [6], Knee Injury and Osteoarthritis Outcome Score (KOOS) [7], and Oxford Knee Score (OKS) [8], are used frequently to evaluate postoperative outcomes after TKA. The Knee Society also revised the evaluation method for TKA to PROMs in 2011, and it was launched as the Knee Society Scoring System 2011 (KSS-2011) [9] [10]. This scoring system includes questionnaires related to the category of satisfaction, which directly evaluates patient satisfaction after TKA. Furthermore, questionnaires about the condition of the knee when more active patients enjoy leisure activities and sports were included in addition to daily activities.

The Forgotten Joint Score-12 (FJS-12) is a PROM developed in 2012 [11]. Good joints are considered "no awareness," and the FJS-12 is useful to evaluate joint awareness. FJS-12 is correlated with PROMs 
associated with knee joints, such as WOMAC, KOOS, and OKS [11-15], and it is effective for evaluating outcomes after TKA. The FJS-12 has an advantage in that it has 12 questions and fewer ceiling and floor effects. However, the relationship between FJS-12 and KSS-2011, which are the most recently developed PROMs used to evaluate postoperative TKA performance, has not been validated. In particular, because KSS-2011 is the only PROM that contains a "patient satisfaction" category, the relationship between FJS12 and subcategories of KSS-2011 including "patient satisfaction" should be evaluated.

In addition, joint noise frequently occurs after TKA. Nam et al. [16] reported that noise after TKA is related to residual symptoms, whereas Kuriyama et al. [17] reported that noise was not correlated with patient satisfaction after TKA. Noise may be related to joint awareness after TKA. However, not enough information is available on whether the noise after TKA is related to patient-reported outcomes including joint awareness.

The aim of this study is to reveal how the joint awareness correlate with knee symptoms, functions, postoperative satisfaction, and noise perception after TKA. To assess this questions, the relationship between FJS-12 and KSS-2011, and the perception of noise was evaluated. Furthermore, the relationship between PROMs (FJS-12, KSS-2011, and noise perception) and factors, such as TKA mechanism and range of movement (ROM), was assessed.

\section{Methods}

A total of 476 patients (598 knees) who underwent primary TKA from January 2007 to November 2017 at our hospital and related institutions and for whom $\geq 1$ year had elapsed after TKA were enrolled in this study. After excluding patients who died or whose implants were removed due to loosening or infection, the questionnaire survey, including FJS-12 and KSS-2011, was mailed to 451 patients (566 knees). We used validated Japanese versions of the questionnaires and signed license agreements with the copyright owners.

In addition, the noise of the knee after TKA was assessed via a questionnaire asking if the patients feel any noise during the activity of daily life. Noise scoring was defined according to a 5-point scale: never feel (4 points), feel almost never (3 points), feel seldom ( 2 points), feel sometimes ( 1 point), and feel mostly (0 points). This questionnaire used the same expression as the FJS-12. In patients who underwent bilateral surgery, responses for two knees on each side were obtained. Valid responses for FJS-12, KSS, and noises were obtained from 289 patients (372 knees, questionnaire collection rate, 65.7\%).

Based on medical records, age at operation, body mass index (BMI) at the time of the survey, and preoperative and postoperative ROM were examined. As a result, 225 (295 knees) patients whose valid data on all items of FJS-12, KSS2011, noise, preoperative ROM, and postoperative ROM were obtained. The present study was approved by the institutional review board of our institution (approval number: 4681).

The 11 models of TKA implant used in this research were classified based on the mechanism as posterior stabilized (PS; 91), cruciate retaining (CR; 78), cruciate sacrificed (which is without post-cam mechanism) 
(CS; 102), and bi-cruciate stabilized (BCS; 24). The models used were Legion ${ }^{\circledR}$ PS (39 knees; Smith \& Nephew, Memphis, TN, USA), Legion ${ }^{\circledR}$ CR (11 knees; Smith \& Nephew),NexGen ${ }^{\circledR}$ LPS (31 knees; Zimmer, Warsaw, IN, USA), NexGen ${ }^{\circledR}$ CR (12 knees; Zimmer) , Genesis II ${ }^{\circledR}$ PS (19 knees; Smith \& Nephew), Genesis $I^{\circledR} \mathrm{CR}$ (31 knees; Smith \& Nephew) , Persona ${ }^{\circledR}$ CR (12 knees; Zimmer), Advance ${ }^{\circledR}$ CR (14 knees; Wright, Memphis, TN, USA), LCS ${ }^{\circledR}$ rotation platform CS (81 knees; Depuy), GMK Sphere ${ }^{\circledR}$ CS (19 knees; Medacta, Strada Regina, Switzerland), and Journey $\|^{\circledR}$ BCS (24 knees; Smith \& Nephew). The TKA models were chosen by the surgeons' preferences at operation.

\section{Statistical analysis}

For statistical examination, Cronbach's a was used to evaluate the internal consistency of FJS-12, and $>0.9$ indicated that the scale is reliable. The Shapiro-Wilk test was used to evaluate normal distribution. The ceiling effect was defined when it satisfied $\geq 85 \%$ of the maximum score and the floor effect satisfied $\leq 15 \%$ of the maximum score. Because the Shapiro-Wilk test showed that FJS-12 and KSS-2011 did not show normal distribution, the correlation between FJS-12 and KSS-2011 was examined using Spearman's correlation coefficient. Noise was examined also for correlation with FJS-12 or KSS-2011. In addition, FJS$12, \mathrm{KSS}-2011$, and noise examined the correlation between the patient characteristics (age at operation and $\mathrm{BMI}$ at questionnaire collection), and ROM before and postoperatively. Regarding the TKA mechanism, differences among the mechanisms underlying patient characteristics, ROM, FJS-12, KSS2011, and noise were examined using analysis of variance (ANOVA). In relation to ANOVA, power analysis was performed and the detection power was calculated. Multiple comparisons were performed with the Steel-Dwass test.

JMP Pro 14.0.0 was used for statistical processing, and the rejection area was set to $5 \%$.

\section{Results}

Table 1 shows the demographic data for subject characteristics.

FJS-12 had an average score of 49.8 [standard deviation (SD), 28.4]. The ceiling effect was $15.6 \%$, and the floor effect was $11.9 \%$ (Table 2). Regarding the average value of each of the 12 items, question 8 ("when you are standing up from a low-sitting position?") had the worst score (average, 3.67 points). Regarding response rate, several patients (14.5\%) did not answer question 12 ("when you are doing your favorite sport?";Table 3). The value for Cronbach's a was 0.947.

KSS-2011 had an average of 119.3 (SD, 32.4), and the ceiling effect, floor effect, and the results of each item are shown in Tables 2 and 4.

A strong correlation was observed between FJS-12 and the total scores of KSS-2011 $(0.70 ; 0.65-0.75 ; P<$ 0.001 in $95 \%$ confidence interval; Fig.1). Table 4 shows the correlation between FJS-12 and the subscores of KSS-2011 by category. A positive correlation was observed in all items, and correlation coefficients of 
approximately 0.60 were observed for "symptoms," "patient satisfaction," "functional activities," and "standard activities."

Noise showed weak correlations with FJS-12 $(0.28 ; P<0.001)$ and KSS-2011 $(0.20 ; P<0.001)$. Age, BMI, and ROM had no effect on FJS-12, KSS-2011, and noise (Table 5).

Moreover, FJS-12, KSS-2011, postoperative ROM, and noise were examined according to the mechanisms (CS, CR, PS, and BCS) and no significant differences were observed in FJS-12. However, a significant difference was observed in KSS-2011, postoperative ROM, and noise. BCS had the best performance among the four mechanisms in KSS-2011 and postoperative ROM, while it had the lowest score in noise. Multiple comparisons revealed that BCS showed significantly better KSS-2011 scores than PS, greater ROM than most of the other mechanisms, but lower noise score than CR (Table 6).

\section{Discussion}

This study revealed that FJS-12 and KSS-2011 had a strong correlation in patients who underwent TKA, confirming the validation of the two most recent scoring system with each other. Although noise was hypothesized to have an effect on joint awareness, only a weak correlation was observed with FJS-12 and KSS-2011. In fact, BCS showed the worst score in noise, but the best score in KSS-2011 and the greatest ROM. Noise is suggested to have a limited effect on joint awareness and clinical outcomes. Residual symptoms and joint functions rather than noise perception might be important for joint awareness after TKA.

Of the subscores of KSS-2011, correlation coefficients of approximately 0.60 were observed for "symptoms," "patient satisfaction," and "functional activities" for FJS-12. It became clear that joint awareness was correlated with postoperative pain and activities of daily living. Although FJS-12 does not directly question patient satisfaction, it also can evaluate patient satisfaction because it also is associated with patient satisfaction from KSS-2011, which directly asks for satisfaction. In addition, as the functional activities of KSS-2011 are divided into subcategories ("walking and standing," "standard activities," "advanced activities," and "discretional activities"), our study indicated a significant correlation between FJS-12 and standard activities. Therefore, joint awareness was influenced by the difficulty level of routine activities, such as walking on an uneven surface, turning or pivoting, climbing up or down stairs, rising from a low chair, and stepping to the side. FJS-12 is a simple questionnaire with only 12 items and has few ceiling and floor effects. Therefore, FJS-12 is a useful tool to evaluate residual symptoms, performance of daily activities, and patient satisfaction after TKA.

Noise was expected to be correlated with joint awareness. However, it had only a weak correlation with FJS-12 and KSS-2011. Although patients with TKA are aware of noise, some studies have shown that it has minimal influence on patient satisfaction if the functional aspects, such as ROM, are good [17]. This previous study was done with a TKA model using a unique tri-condylar mechanism. Therefore, the influence of noise perception on PROMs for patients with conventional TKA models needed to be elucidated. In the current study of TKA mechanisms, a significant difference was observed between KSS- 
2011 and postoperative ROM, and the BCS results were good. A significant difference was confirmed regarding noise, and the BCS score was the lowest. Nam et al. [16] reported that noise was recognized in $27 \%$ of the patients and the likelihood of noise generation was different among the TKA mechanisms; PS design was the greatest, followed by rotating-platform, sex-specific, and CR factors. Furthermore, patientrecognized noise reportedly was associated with residual symptoms, such as difficulty in getting in and out of cars, limp, stiffness, or swelling. The inconsistency between the previous studies and our study can be caused by how to assess the noise frequency. The previous studies categorized subjects into two groups according to with or without noise, while the current study used an ordinal scale. From our findings, even if noise was recognized, patient satisfaction postoperatively would be high if functions, such as the ROM, were good. Therefore, noise itself had a limited effect on joint function, overall satisfaction, and joint awareness after TKA.

The present study had several limitations. First, there was a question with a low response rate in FJS-12. As reported in previous studies [18] [19], the response rate for item 12 was particularly low. It may be reasonable to consider that some patients who underwent TKA were relatively inactive. However, FJS-12 has been confirmed to be valid with a few missing items [11].

Second, this was a retrospective cross-sectional study, and there were variations in the time when the answers for FJS-12 were collected. In fact, some studies have shown that the FJS-12 scores change over time [20]. The differences in questionnaire sampling time might have influenced the results. However, we recruited patients for whom at least 1 year had passed postoperatively.

Third, various TKA models had been used. Although the mechanisms can be categorized into four systems, several models remained within the same category. Furthermore, preoperative patient conditions were not matched among the groups (Table 6). In fact, preoperative ROM was greater in BCS. Therefore, this study did not conclude that BCS was superior in clinical outcomes than any other TKA mechanisms. We did not attempt to investigate which mechanism had the best performance. The point of this analysis was to show that some TKA model exhibit greater noise generation while showing higher KSS-2011 score. Thus, this finding suggested that noise perception has a limited effect on clinical outcomes.

Lastly, the noise score used in this study has not been validated before. Because there is no score as a reference to evaluate the noise perception, the same questionnaire expression method as the FJS-12 was used to evaluate noise.

\section{Conclusions}

A strong correlation was observed between FJS-12 and KSS-2011. In addition, FJS-12 was associated with the subcategories of KSS-2011 for symptoms, patient satisfaction, and standard activities. Noise did not have a significant association with joint awareness or clinical outcomes as assessed by FJS-12, KSS2011, or ROM. Some knees exhibited greater postoperative ROM and KSS-2011 with more frequent noise perception. 


\section{Abbreviations}

BCS: Bi-cruciate Stabilized, BMI: Body Mass Index, CR: Cruciate Retaining, CS: Cruciate Sacrificed, FJS-12: Forgotten Joint Score - 12, KSS-2011: 2011 Knee Society Knee Scoring System, KOOS: Knee Injury and Osteoarthritis Outcome Score, OKS: Oxford Knee Score, PROMs: Patient-reported Outcome Measures, PS: Posterior Stabilized, ROM: Range of Motion, S.D.: Standard Deviation, S.E.: Standard Error, TKA: Total Knee Arthroplasty, WOMAC: the Western Ontario and McMaster Universities Osteoarthritis Index.

\section{Declarations}

\section{Ethics approval and consent to participate}

This study was approved by the Medical Ethical Committee of the Tokyo Women's Medical University (approval number: 4681). The form of Informed consent in this study is opt-out.

\section{Consent for publication}

Not applicable.

\section{Availability of data and materials}

Data are attached to supplementary material as an Excel document.

\section{Competing interests}

The authors declare that they have no competing interests, except KO is a paid speaker of Smith and Nephew, inc. and Zimmer Biomet, inc.

\section{Funding}

Not applicable.

\section{Authors' contributions}

$\mathrm{KO}$ and $\mathrm{HT}$ designed the study and completed the draft of the manuscript. All authors made contributions to the conception and outline of the study. HT, MI, NY, JI and UK collected and analyzed of the data of PROMs. Especially, MI and HT collected and analyzed of the data of noise. $\mathrm{KO}$ and $\mathrm{HT}$ contributed to the interpretation of the data. KO contributed to writing and editing, as well as reviewed the manuscript. All authors read and approved the final manuscript.

\section{Trial registration}

This study was approved by the Medical Ethical Committee of the Tokyo Women's Medical University (approval number: 4681 on March 2, 2018). 


\section{Acknowledgements}

Not applicable.

\section{Author's information}

Nothing.

\section{References}

1. Robinson, R. P. (2005). The early innovators of today's resurfacing condylar knees. The Journal of Arthroplasty, 20, 2-26. https://doi.org/10.1016/j.arth.2004.11.002

2. Matsuda, S., Kawahara, S., Okazaki, K., Tashiro, Y., \& Iwamoto, Y. (2013). Postoperative alignment and ROM affect patient satisfaction after TKA. Clinical Orthopaedics and Related Research $\Theta, 471(1)$, 127-133. https://doi.org/10.1007/s11999-012-2533-y

3. Wylde, V., Blom, A. W., Whitehouse, S. L., Taylor, A. H., Pattison, G. T., \& Bannister, G. C. (2009). Patientreported outcomes after total hip and knee arthroplasty: comparison of midterm results. The Journal of Arthroplasty, 24(2), 210-216. https://doi.org/10.1016/j.arth.2007.12.001

4. Marx, R. G., Jones, E. C., Atwan, N. C., Closkey, R. F., Salvati, E. A., \& Sculco, T. P. (2005). Measuring improvement following total hip and knee arthroplasty using patient-based measures of outcome. The Journal of Bone and Joint Surgery. American volume, 87(9), 1999-2005.

https://doi.org/10.2106/JBJS.D.02286

5. Bourne, R. B., Chesworth, B., Davis, A., Mahomed, N., \& Charron, K. (2010). Comparing patient outcomes after THA and TKA: is there a difference? Clinical Orthopaedics and Related Research ${ }^{B}$, 468(2), 542-546. https://doi.org/10.1007/s11999-009-1046-9

6. Bellamy, N., \& Buchanan, W. W. (1986). A preliminary evaluation of the dimensionality and clinical importance of pain and disability in osteoarthritis of the hip and knee. Clinical Rheumatology, 5(2), 231-241.

7. Roos, E. M., Roos, H. P., Lohmander, L. S., Ekdahl, C., \& Beynnon, B. D. (1998). Knee Injury and Osteoarthritis Outcome Score (KOOS)-development of a self-administered outcome measure. Journal of Orthopaedic \& Sports Physical Therapy, 28(2), 88-96. https://doi.org/10.2519/jospt.1998.28.2.88

8. Dawson, J., Fitzpatrick, R., Carr, A., \& Murray, D. (1998). Questionnaire on the perceptions of patients about total knee replacement. The Journal of Bone and Joint Surgery. British volume, 80(1), 63-69.

9. Scuderi, G. R., Bourne, R. B., Noble, P. C., Benjamin, J. B., Lonner, J. H., \& Scott, W. N. (2012). The new knee society knee scoring system. Clinical Orthopaedics and Related Research ${ }^{\circledR}, 470(1), 3-19$. https://doi.org/ 1007/s11999-011-2135-0 
10. Noble, P. C., Scuderi, G. R., Brekke, A. C., Sikorskii, A., Benjamin, J. B., Lonner, J. H., \& Bourne, R. B. (2012). Development of a new Knee Society scoring system. Clinical Orthopaedics and Related Research®, 470(1), 20-32. https://doi.org/10.1007/s11999-011-2152-z

11. Behrend, H., Giesinger, K., Giesinger, J. M., \& Kuster, M. S. (2012). The "forgotten joint" as the ultimate goal in joint arthroplasty: validation of a new patient-reported outcome measure. The Journal of Arthroplasty, 27(3), 430-436. https://doi.org/10.1016/j.arth.2011.06.035

12. Cao, S., Liu, N., Han, W., Zi, Y., Peng, F., Li, L.,... \& Qian, Q. (2017). Simplified Chinese version of the Forgotten Joint Score (FJS) for patients who underwent joint arthroplasty: cross-cultural adaptation and validation. Journal of Orthopaedic Surgery and Research, 12(1), 6.

https://doi.org/10.1186/s13018-016-0508-5

13. Thomsen, M. G., Latifi, R., Kallemose, T., Barfod, K. W., Husted, H., \& Troelsen, A. (2016). Good validity and reliability of the forgotten joint score in evaluating the outcome of total knee arthroplasty: $A$ retrospective cross-sectional survey-based study. Acta Orthopaedica, 87(3), 280-285. https://doi.org/10.3109/17453674.2016.1156934

14. Giesinger, J. M., Kuster, M. S., Behrend, H., \& Giesinger, K. (2013). Association of psychological status and patient-reported physical outcome measures in joint arthroplasty: a lack of divergent validity. Health and Quality of Life Outcomes, 11(1), 1. https://doi.org/10.1186/1477-7525-11-64

15. Giesinger, J. M., Loth, F. L., MacDonald, D. J., Giesinger, K., Patton, J. T., Simpson, A. H. R. W., \& Hamilton, D. F. (2018). Patient-reported outcome metrics following total knee arthroplasty are influenced differently by patients' body mass index. Knee Surgery, Sports Traumatology, Arthroscopy, 1-8. https://doi.org/10.1007/s00167-018-4853-2

16. Nam, D., Barrack, T., Nunley, R. M., \& Barrack, R. L. (2017). What is the frequency of noise generation in modern knee arthroplasty and is it associated with residual symptoms? Clinical Orthopaedics and Related Research®, 475(1), 83-90. https://doi.org/10.1007/s11999-016-4701-y

17. Kuriyama, S., Ishikawa, M., Nakamura, S., Furu, M., Ito, H., \& Matsuda, S. (2017). Noise generation with good range of motion but without femorotibial instability has small effect on patient satisfaction after total knee arthroplasty. The Journal of Arthroplasty, 32(2), 407-412. https://doi.org/10.1016/j.arth.2016.07.012

18. Shadid, M. B., Vinken, N. S., Marting, L. N., \& Wolterbeek, N. (2016). The Dutch version of the Forgotten Joint Score: test-retesting reliability and validation. Acta Orthop Belg, 82(1), 112-118. https://www.ncbi.nlm.nih.gov/pubmed/26984663

19. Thienpont, E., Opsomer, G., Koninckx, A., \& Houssiau, F. (2014). Joint awareness in different types of knee arthroplasty evaluated with the Forgotten Joint score. The Journal of Arthroplasty, 29(1), 48-51. https://doi.org/10.1016/j.arth.2013.04.024

20. Giesinger, K., Hamilton, D. F., Jost, B., Holzner, B., \& Giesinger, J. M. (2014). Comparative responsiveness of outcome measures for total knee arthroplasty. Osteoarthritis and Cartilage, 22(2), 184-189. https://doi.org/10.1016/j.joca.2013.11.001 


\section{Figures}

Figure 1.

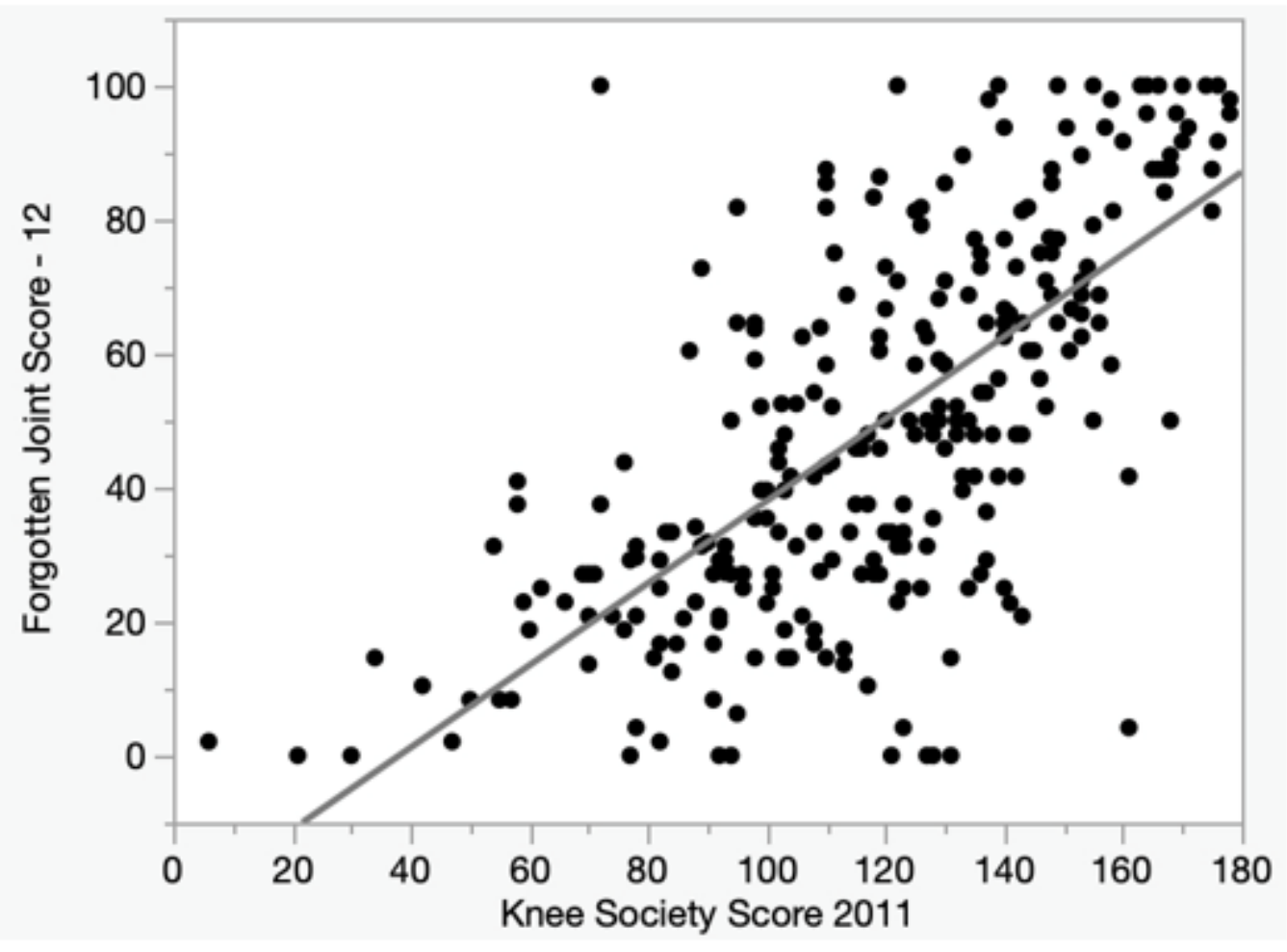

Figure 1

The graph shows the relationship between Forgotten Joint Score-12 (FJS-12) and the Knee Society Scoring System-2011 (KSS-2011) score. Linear regression analysis showed a strong correlation between FJS-12 and KSS-2011 scores with a correlation coefficient of 0.70 ( $95 \%$ confidence interval, $0.65-0.75 ; \mathrm{P}$ $<0.001)$.

\section{Supplementary Files}

This is a list of supplementary files associated with this preprint. Click to download.

- Tables.pdf 